\title{
DEFECTS AND THEIR ORIGIN IN THIN FILMS OF (001) ALKALINE EARTH OXIDES
}

\author{
F. J. WALKER*, R. A. MCKEE, S. J. PENNYCOOK AND T. G. THUNDAT \\ Oak Ridge National Laboratory, Metals and Ceramics Division, Oak Ridge, TN \\ $37831-6118$ \\ *Also associated with the Department of Materials Science and Engineering, \\ University of Tennessee, Knoxville, TN
}

\begin{abstract}
$\mathrm{MgO}$ is used as an optical isolation layer for waveguides epitaxially grown on silicon. The crystalline perfection of $\mathrm{MgO}$ is a critical issue because it serves as a substrate for the single crystal, perovskite guiding layer. Imperfections in the $\mathrm{MgO}$ will result in imperfections in the guiding layer and lead to large optical losses for the planar waveguide structure. We show that the most common defect to form in thin films of $\mathrm{MgO}$ are twin boundaries between \{111\}-type planes. The highest density of twins is observed when (001) $\mathrm{MgO}$ is grown directly on silicon/MgO interlayers containing barium. Twinning is shown to accommodate the large size of barium impurities incorporated in the $\mathrm{MgO}$ films through the formation of internal grain boundaries and open surfaces other than the growing (001) of $\mathrm{MgO}$.
\end{abstract}

\section{INTRODUCTION}

Our interest in alkaline earth oxides is driven in part by thin film, optical waveguide applications [1]. For many thin film applications, the ideal substrate is silicon. For applications that require a large and fast electro-optic coefficient, the ideal guiding layer is $\mathrm{BaTiO}_{3}$. In order to make the structure guide light, however, a third layer is needed in between the $\mathrm{BaTiO}_{3}$ and the silicon to avoid coupling the light out of the guide. We have chosen $\mathrm{MgO}$ for this layer because of its relative stability in air against hydration, its simplicity of deposition and its small index of refraction $(n=1.74)$.

Of the current applications of thin film oxides, optical waveguides place perhaps the most stringent requirements on film perfection. After the inception of integrated optics using thin films, the first recognized materials problem was surface roughness. Tien showed the importance theoretically and experimentally of this parameter in his work on $\mathrm{ZnO}$ films [2]. These films exhibited a large optical transmission loss due to light being scattered out of the guide by surface roughness. This roughness is largely a result of faceting of polycrystalline crystallites at the surface. This problem has resurfaced and been studied in detail in more recent work by Fork et al. [3] on epitaxial thin films grown for second harmonic generation. Practical, integrated optics applications will require even higher degrees of crystalline perfection to avoid even a single scattering defect in tens of micrometer-long channel guides that are 1 to $5 \mu \mathrm{m}$ wide. For waveguide structures on silicon, this perfection must be maintained in the isolation layer throughout a deposition of up to $1 \mu \mathrm{m}$ thick.

In addition to application driven interest, defects, surfaces and interfaces of alkaline earth oxides and $\mathrm{MgO}$ have been the focus of atomistic theoretical investigations. Investigators at Harwell and D. Wolf at Argonne National Laboratory have calculated the energetics and stability of point defects [4], low angle grain boundaries [5], twin boundaries [6], stacking faults and twins [6], segregation of impurities at surfaces [7], 
atomic structure at surface steps [8] and interfaces between alkaline earth oxides [9]. One motivation for this theoretical work is the application of the results to the sintering of oxide materials [5]. For this application, it was not envisioned that experimental results of sintering would test the theory, but that the theory would provide a fundamental understanding of the basic processes. The reason is that sintering is a complex process and separating the contribution of competing processes is not practical. Surfaces and thin film structures, however, represent a simpler system and have been used to test the applicability of these theoretical calculations to defect diffusion in $\mathrm{MgO}$ [10]. Once the applicability of a method of calculation has been demonstrated, the experiments can then be extrapolated using atomistic calculations to determine the optimum materials combination for thin film structures that are highly perfect and optically clear.

For optical waveguide applications, we have grown $\mathrm{MgO}$ with a (001) orientation on silicon. As described below, single crystal $\mathrm{MgO}$ will not grow directly on silicon, so that an oxide buffer layer is required between the $\mathrm{MgO}$ and silicon. For example, $\mathrm{BaO}$ is an oxide that grows well on (001) $\mathrm{Si}$ [11]. The (001) surface of $\mathrm{MgO}$ is well suited for waveguide applications. We have shown that $\mathrm{BaTiO}_{3}$ can be grown on this surface with optical clarity [1]. Most of the alkaline earth oxides ( $\mathrm{BaO}, \mathrm{SrO}, \mathrm{CaO}$ and $\mathrm{MgO}$ ) are ionic and have the rock salt crystal structure. The structure and the ionicity combine to make the $\{001\}$-type surfaces highly stable. The next most stable surfaces of $\mathrm{MgO}$, the $\{110\}$-type, have a specific energy of $2.79 \mathrm{~J} / \mathrm{m}^{2}$ compared to $1.06 \mathrm{~J} / \mathrm{m}^{2}$ for the energy of the $\{001\}$-type surfaces [7]. Our previous experience with deposition of thick films of $\mathrm{BaO}(001)$ on $\mathrm{Si}(001)$ led us to believe that all AEO (001) surfaces will allow single crystal growth at low temperatures $\left(<275^{\circ} \mathrm{C}\right)$ and high deposition rates ( $>10 \AA / \mathrm{sec}$ ) [11]. As we will describe in this paper, however, growth on the $\mathrm{MgO}(001)$ would often twin.

\section{EXPERIMENTAL AND RESULTS}

All of the thin films we describe have been grown in an ultra high vacuum (UHV) molecular beam epitaxy (MBE) growth chamber [12]. We grow all of the oxides except $\mathrm{MgO}$ by reacting the metals with oxygen leaked into the growth chamber at pressures from $1 \times 10^{-7}$ Torr to $1 \times 10^{-5}$ Torr. An electron beam source is used to deposit $\mathrm{MgO}$ at pressures of around $5 \times 10^{-9}$ Torr to $1 \times 10^{-8}$ Torr. Mass spectroscopy reveals that most of this pressure can be attributed to hydrogen. $\mathrm{MgO}$ will not grow directly on silicon with a high degree of crystalline quality but will grow with more than one epitaxial alignment. The reason is the large misfit between $\mathrm{MgO}$ and the $\mathrm{Si}(001)$ surface. The smallest misfit is $8.8 \%$ for $\mathrm{MgO}$ growing with (001)MgO $\|(001) \mathrm{Si}$ and aligned so that $\langle 110\rangle \mathrm{MgO} \|\langle 100\rangle \mathrm{Si}$. For alignments of the $\mathrm{MgO} 45^{\circ}$ rotated from this minimum misfit, the misfit is $29 \%$. To avoid this large misfit we grow a variety of oxide buffer layer structures (see Table I) all of which minimize the misfit at the silicon interface.

Epitaxial growth of $\mathrm{MgO}$ (001) becomes twinned after deposition of just nm's to 100 's of nms of $\mathrm{MgO}$. The thickness at which diffraction from twins is observed depends on the buffer layer. In Fig. 1 we have reproduced a reflection high energy electron diffraction (RHEED) pattern from the (001) $\mathrm{MgO}$ surface that exhibits twinned growth. In this pattern, the electron beam was directed down the $\langle 110\rangle$ zone axis of the predominately (001) surface of the $\mathrm{MgO}$ film. The extra spots are attributed to twin crystallites. The pattern defined by the extra spots is consistent with diffraction from a [110] zone axis of $\mathrm{MgO}$ where the [111] direction is common to both the twinned 


\begin{tabular}{|l|c|c|c|}
\hline Substrate & $\begin{array}{c}\text { Propen- } \\
\text { sity to } \\
\text { twin }\end{array}$ & $\begin{array}{c}\text { Thickne- } \\
\text { ss twins } \\
\text { observed }\end{array}$ & $\begin{array}{c}\text { Misfit } \\
\text { with } \\
\mathrm{MgO}\end{array}$ \\
\hline $\mathrm{BaO}$ & strong & 10 's of $\mathrm{nm}$ & $-7.2 \%$ \\
\hline $\mathrm{CaTiO}_{3}$ & weak & $\begin{array}{c}100 \text { 's of } \\
\mathrm{nm}\end{array}$ & $-7.7 \%$ \\
\hline $\mathrm{BaO}$ to $\mathrm{CaO}$ & moderate & $100 \mathrm{~nm}$ & $12.9 \%$ \\
\hline $\mathrm{BaTiO}_{3}$ & strong & $\mathrm{nm}$ 's & $-3.7 \%$ \\
\hline Dirty $\mathrm{MgO}$ & weak & 10 's of nm & $0 \%$ \\
\hline
\end{tabular}

Table I. Summary of propensity of $\mathrm{MgO}$ growth to twin on when grown on various buffer layers.

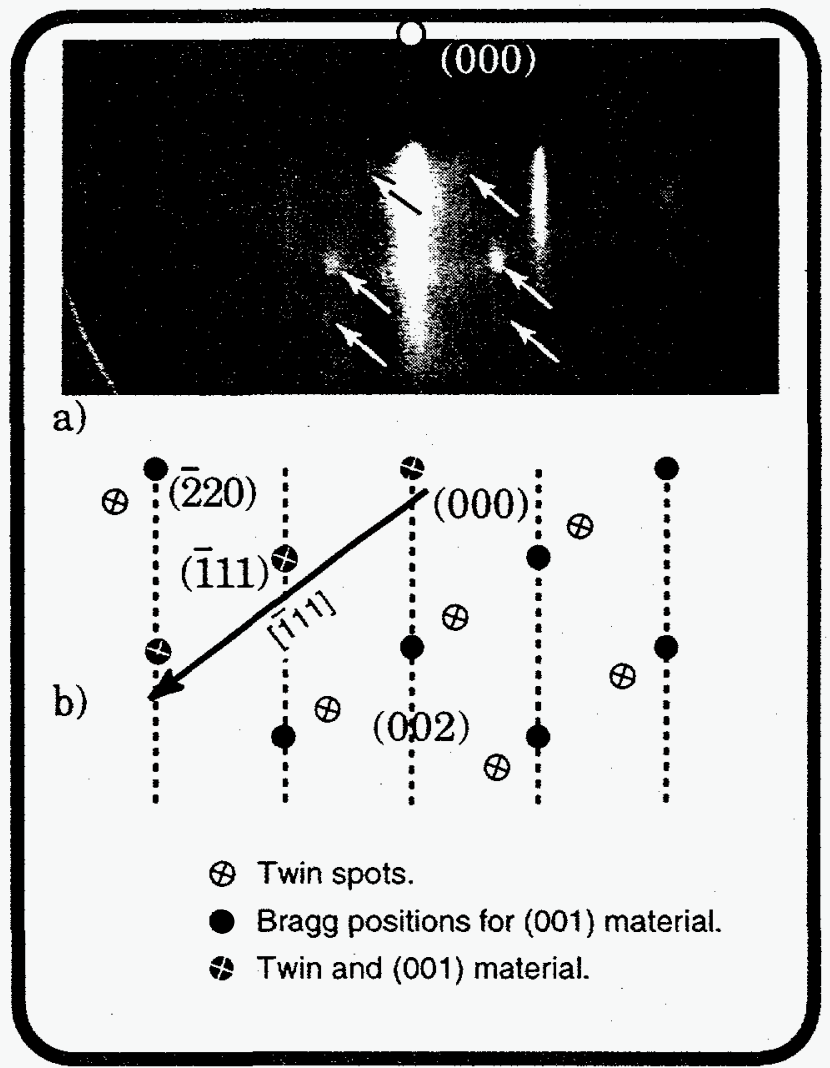

Figure 1. a) RHEED pattern showing 3D diffraction due to twin. b) Simulated RHEED pattern. The spots indexed in the simulation are for the (001) MgO. Dashed lines show positions of diffraction rods from the (001) surface of MgO. material and the (001) growing $\mathrm{MgO}$. The simulation of the twin pattern can be derived from the (001) $\mathrm{MgO}$ pattern by a rotation of $180^{\circ}$ about the [111] direction. The arrows in Fig. 1 mark the positions of the twin spots for two twin variants. Half of these diffraction spots correspond to the light circles with crosses in the diffraction pattern simulation shown in Fig. 1. The other half of the spots indicated by arrows in the RHEED not accounted for by the simulation are from the second variant. The diffraction for this variant is related to the twin of the simulation by a mirror operation along the (002) $\mathrm{MgO}$ direction. This is the variant for twinning on the (111) plane. In addition to the orientation, the diffraction also reveals that the twinned material is not flat and protrudes from the flat surface of the (001) $\mathrm{MgO}$. This follows from the fact that diffraction from the (001) MgO is in the form of rods perpendicular to the film surface whereas diffraction from the twinned material is a spot. Rods of diffraction arise from the two dimensional nature of electron scattering from a flat surface where the penetration depth of the electrons is limited by the small incident angle of the electrons. Bragg spots arise from the three dimensional nature of scattering through a small crystallite protruding from the surface.

We can characterize the propensity of $\mathrm{MgO}$ to twin on different substrates by noting the deposited thickness at which the twin spots are observed. The thickness at which twinning is first observed should be roughly proportional to the density of twins that form on the growing surface. The results of these observations are summarized in Table I for various buffer layers. In addition to the obvious differences in composition, the inplane lattice parameters of the (001) surfaces also vary from $5.53 \AA$ for $\mathrm{BaO}, 4.02$ $\AA$ for $\mathrm{BaTiO}_{3}, 4.81 \AA$ for $\mathrm{CaO}$ and $3.80 \AA$ for $\mathrm{CaTiO}_{3}$. The propensity to twin is not 
a)

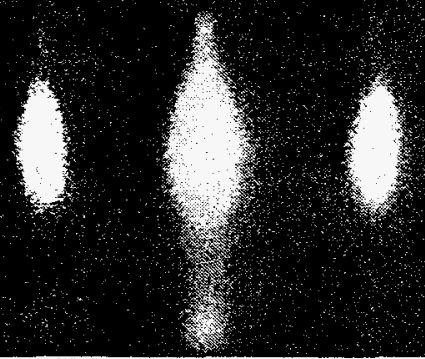

b)

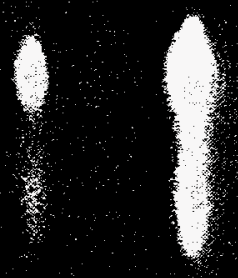

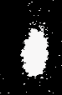

Figure 2. RHEED from a) $60 \mathrm{~nm}$ thick film of $\mathrm{MgO}$ grown on $\mathrm{BaO}: \mathrm{SrO}$ alloy at $500^{\circ} \mathrm{C}$. b) Same film annealed to $800^{\circ} \mathrm{C}$.

strongly correlated to lattice mismatch as seen in Table I. Growth on $\mathrm{CaTiO}_{3}$, for example, shows the least tendency to twin but has a larger misfit with $\mathrm{MgO}$ than does $\mathrm{BaTiO}_{3}$, for which the propensity to twin is high. Instead, the results summarized in Table I indicate that the presence of barium is correlated with the propensity to twin.

Alloying and surface segregation of barium in $\mathrm{MgO}$ has been observed by RHEED and ESCA. Figure 2 is a RHEED pattern from an MgO film grown on $\mathrm{BaO}: \mathrm{SrO}$ alloy at a relatively low temperature of $500{ }^{\circ} \mathrm{C}$ and then annealed to $800^{\circ} \mathrm{C}$ to a thickness of $60.0 \mathrm{~nm}$. The electrons were directed down the $\langle 010\rangle$ zone axis of cubic $\mathrm{MgO}$ as is evident by the square pattern of Bragg reflections. As grown, the $\mathrm{MgO}$ surface is not atomically flat and gives spots located at $3 \mathrm{D}$ Bragg reflections. When the film is heated, the pattern is significantly modified in that the pattern of Bragg reflections is no longer square, but rectangular. Further, electron surface chemical analysis (ESCA) shows that barium surface segregates in the annealed sample. Because the RHEED spots are more closely spaced in the vertical direction in the annealed sample, the $\mathrm{MgO}$ lattice is expanded by the barium impurities in the near surface region but constrained by the underlying $\mathrm{MgO}$. The ratio of vertical to horizontal spacing is $15 \%$ and corresponds to a $15 \%$ volume expansion of the $\mathrm{MgO}$ lattice. Such large volume expansions have been observed in the alkali halide alloys by Yang and Flynn [13].

As indicated by the RHEED from the twinned MgO, we expect the twins to be

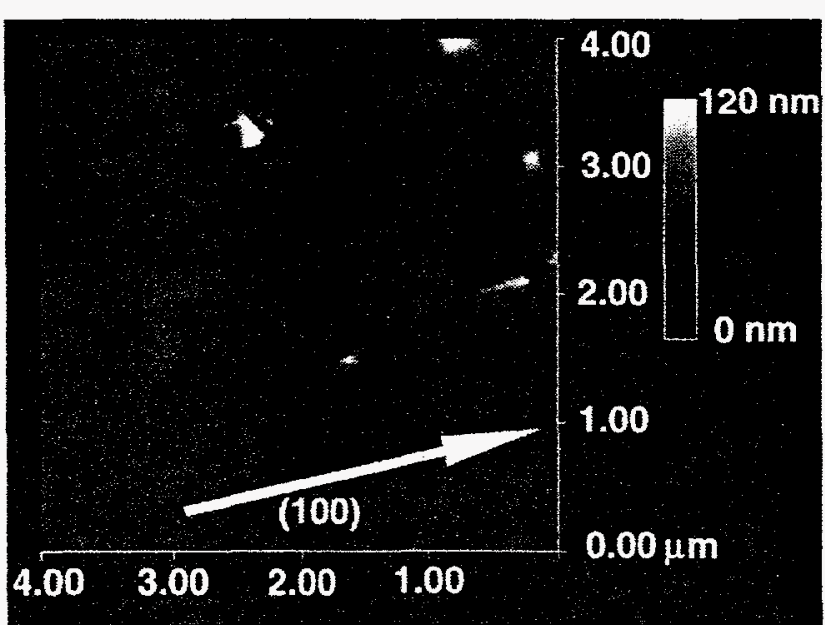

Figure 3. AFM image of $400 \mathrm{~nm}$ thick $\mathrm{MgO}$ surface grown on $\mathrm{CaTiO}_{3}$. The crystal direction shown is that of the predominately (001) $\mathrm{MgO}$. Rms roughness of flat area is $8 \AA$. crystallites protruding from a flat $\mathrm{MgO}$ (001) surface. Atomic force microscope images from the surfaces of $400 \mathrm{~nm}$ of $\mathrm{MgO}$ grown from $\mathrm{CaTiO}_{3}$ surfaces are shown in Figure 3. The measured rms roughness of the (001) surface alone is $8 \AA$. The faceted crystallites we associate with twinned $\mathrm{MgO}$ evident in Figure 3 have no predominate shape. If growth is interrupted earlier at 100 $\mathrm{nm}$, a more regular triangular crystallite is observed protruding from the surface as shown in the left half of Figure 4. For this thinner film, the (001) $\mathrm{MgO}$ surface is flatter having a rms roughness of $3 \AA$. The simple triangular shape of the twin crystallites 


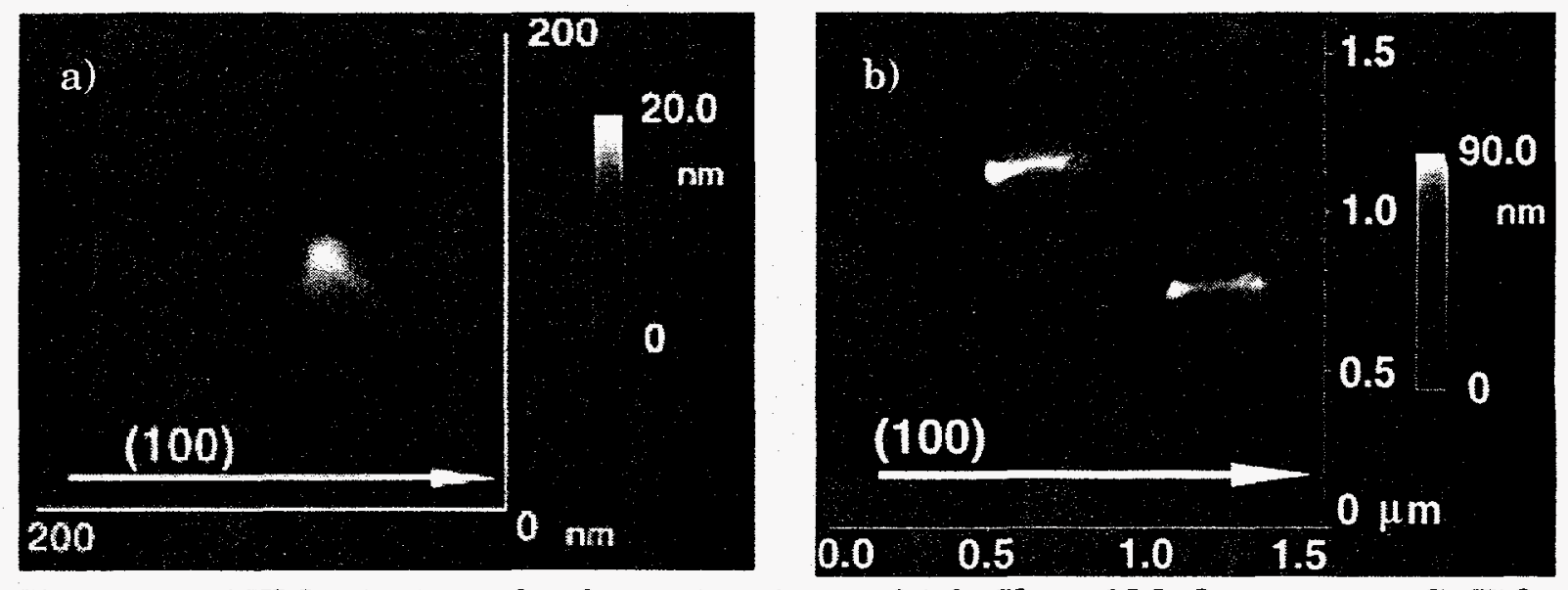

Figure 4. AFM micrographs from a) $100 \mathrm{~nm}$ thick film of $\mathrm{MgO}$ grown on $\mathrm{CaTiO}_{3}$ $\left(\mathrm{rms}\right.$ roughness $=3 \AA$ ) and b) $400 \mathrm{~nm}$ thick film of $\mathrm{MgO}$ grown on $\mathrm{CaTiO}_{3}(\mathrm{rms}$ roughness $=8 \AA$ ). Crystal direction indicated is for the predominate $\mathrm{MgO}$ (001) surface.

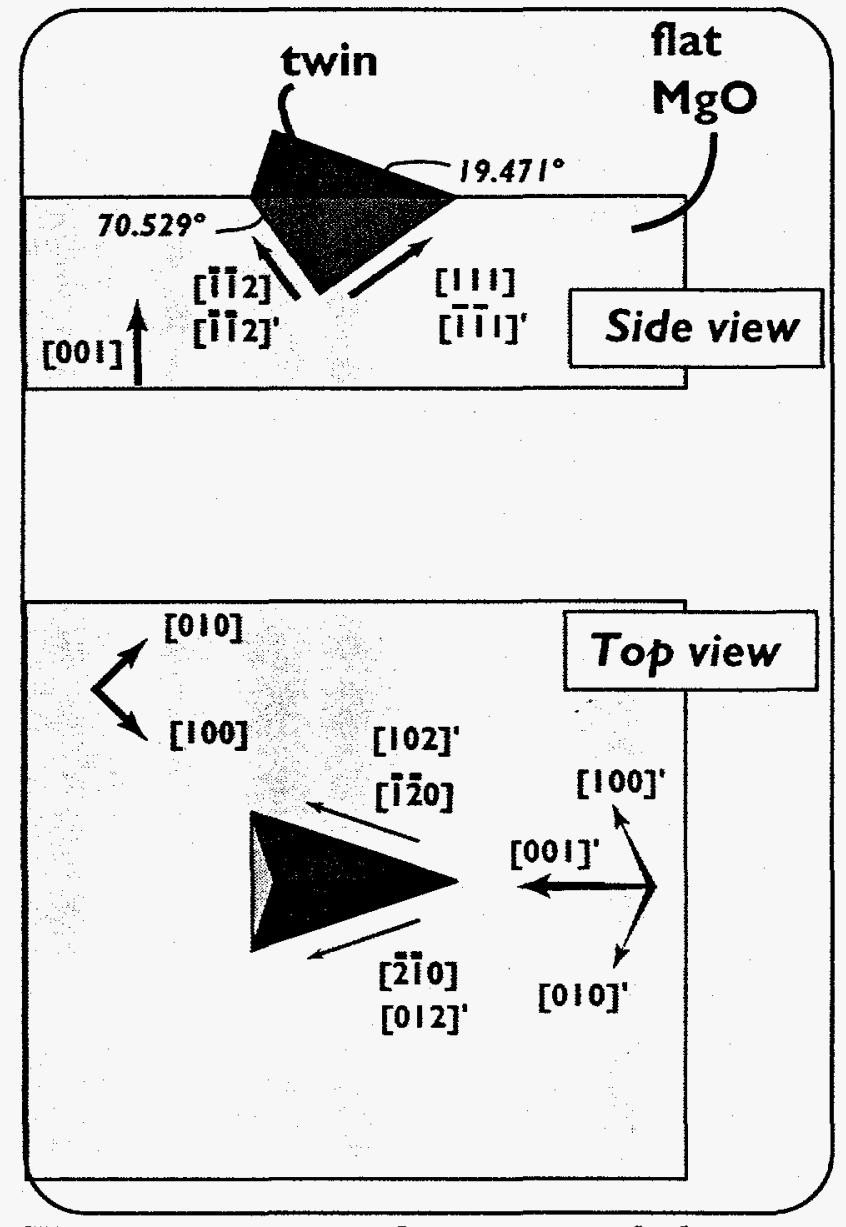

Figure 5. Expected twin morphology on (001) MgO. This morphology is based on the lowest energy surfaces and grain boundaries between the twin and the (001) $\mathrm{MgO}$. Crystallographic directions are primed for the twin and unprimed for the (001) $\mathrm{MgO}$. must evolve by a mechanism more complicated than simply growing larger. This is evident if some of the simpler shapes (right half of Figure 4.) observed on the $400 \mathrm{~nm}$ thick film are compared with the triangular crystallite of the thinner film.

\section{DISCUSSION AND CONCLUSIONS}

The basic problem is that from the measured and theoretically calculated surface energies, the (001) surface of $\mathrm{MgO}$ is extremely stable and the observed twinning is therefore unexpected for pure $\mathrm{MgO}$. As discussed by Yadavalli et al. for rock salt (001) surfaces such as $\mathrm{MgO}$ and the alkali halides [14], growth should occur by simple attachment of $\mathrm{MgO}$ molecules to surface ledges and kinks. Such a mechanism should preserve the (001) orientation.

For the twins that form, we can construct an equilibrium model of the crystallite shape based on the calculated surface and interface energies for pure $\mathrm{MgO}$, and the orientation as observed with RHEED. This model is shown in Figure 5. The vacuum-twin surfaces are simply the stable $\{001\}$-type surfaces. As suggested by the diffraction (see Fig. 1), one of the internal grain boundaries in the 
model is the $\{111\}$ twin. This grain boundary has a low specific energy and has been calculated by Wolf [6] at $0.851 \mathrm{~J} / \mathrm{m}^{2}$. The intersection of the $\{100\}$-type surfaces of the twin inclusion with the (001) $\mathrm{MgO}$ surface define a $\langle 210\rangle$ direction for both the twin and (001) $\mathrm{MgO}$. Possible high symmetry grain boundaries defined by this intersection are specified by:

$$
\text { (211) } \mathrm{MgO} \|(\overline{12} 1) \text { twin with }[20 \overline{4}] \mathrm{MgO} \|[\overline{4} 20] \text { twin }
$$

or, alternatively, by:

$$
(\overline{212}) \mathrm{MgO} \|(100) \text { twin with [101] MgO } \|[0 \overline{1} 1] \text { twin. }
$$

Both grain boundaries are commensurate and we expect them to have specific energies that are on the order of the $\{111\}$ twin.

The proposed twin morphology in Figure 5 is not that measured by AFM for any thickness of $\mathrm{MgO}$ as seen by comparing Figure 5 to the AFM images in Figures 3 and 4. An analysis of the AFM images of Figure 4 reveal that the angles of the larger facets are consistent with the formation of $\{210\}$-type and $\{110\}$-type planes of the twin. If the grain boundaries between the twin and (001) $\mathrm{MgO}$ are simple (i.e. single planes extending into the (001) $\mathrm{MgO}$ ), then the crystallites shown in the AFM images would have grain boundaries of the type $\{210\}$-type and $\{110\}$-type, and not those expected from the equilibrium twin morphology of Figure 5.

This collection of observations can be united by including the results of Tasker et al.'s theoretical calculations of surface energies of barium doped $\mathrm{MgO}$ [7]. They observed that, due to size mismatch, incorporating larger AEO ions in the $\mathrm{MgO}$ lattice cost progressively more energy the larger the AEO ion. Barium showed the largest propensity to segregate out of the $\mathrm{MgO}$ lattice and to a free surface, consistent with our observations of barium segregation in Fig. 2. Moreover, the calculations show that the large size of the barium ion can be accommodated by the MgO lattice by the creation of more open surfaces such as the $\{110\}$-type. With the addition of barium to the (001) surface of $\mathrm{MgO}$ the specific surface energy decreases from $1.06 \mathrm{~J} / \mathrm{m}^{2}$ to $0.00 \mathrm{~J} / \mathrm{m}^{2}$ at $1 / 2$ monolayer coverage. The specific energy of the more open $\{110\}$-type surfaces decreases more rapidly as a function of coverage and becomes the more stable of the two surfaces after around $70 \%$ barium coverage. If accommodation of barium at steps is the important mechanism for stabilizing the $\{110\}$-type, then a continuous range of stepped surfaces would become stable for coverages less than $70 \%$ (see Ref. 4 for a discussion of step energies). The increase in the surface energy due to the addition of steps is compensated by a decrease due to the accommodation of the larger barium ion at the steps. The fact that the surface energies decrease to zero and even negative implies that barium impurities provides a driving force for the creation of new surface area.

The picture we propose for the formation of twins then is the following. Barium is incorporated into the $\mathrm{MgO}$ layer during deposition on substrates containing barium. The large ion size of the barium causes it to segregate to the surface where it stabilizes the formation of stepped surfaces. In order to explain how these stepped surfaces produce twinning, we need to extrapolate Tasker et al.'s results to internal grain boundaries. We propose that internal grain boundaries form that are commensurate but that also provide enough volume expansion to accommodate the large barium ion. The $\{111\}$ twin boundary seems an unlikely candidate. Although it is perhaps the lowest energy grain boundary for $\mathrm{MgO}$, the volume expansion is too small to accom- 
modate barium dopants. Wolf has calculated the volume-expansion per cation-site of the $\{111\}$ twin boundary to be $1 / 10$ th the volume difference between $\mathrm{Ba}^{2+}$ and $\mathrm{Mg}^{2+}[6]$. As suggested by the AFM results, other grain boundaries, like $\{210\}$-type and $\{110\}$ type, may form at the barium doped $\mathrm{MgO}$ surface having a volume expansion large enough to accommodate the segregated barium ions.

Eliminating barium from the silicon $/ \mathrm{MgO}$ interlayer and thus the twinning mechanism has resulted in the development of a successful waveguiding structure with low densities of scattering centers. The success of this development depended on the simple predictions provided by atomistic calculations on the consequences of size driven segregation in AEO's. Direct evidence of our proposed mechanism of twin formation can provide quantitative support for the theoretical calculations. Such evidence can be, perhaps, uniquely obtained through chemical and structural characterization using Z-contrast microscopy [15]. Quantitative information, including grain boundary configuration, structure and dopant content, on a range of doped AEO films can further test the predictions of atomistic calculations of the stability of grain boundaries under varying conditions. The simple thin film geometries that provide a useful experimental realization of atomistic calculations also provide a fundamental understanding of the role of defects and dopants in the thin film growth of this important class of oxides.

\section{ACKNOWLEDGMENTS}

The authors would like to express their appreciation to G. E. Ice and E. D. Specht for their critical reading of the manuscript. Research sponsored jointly by the Laboratory Directed Research and Development Program of Oak Ridge National Laboratory, and by the Division of Materials Sciences and Office of Health and Environmental Research. Oak Ridge National Laboratory is managed by Lockheed Martin Energy Research Corp. for the U.S. Department of Energy under contract DE AC0596OR22464.

\section{REFERENCES}

1. R.A. McKee, F. J. Walker, E. D. Specht, G. E. Jellison, Jr., and L. A. Boatner, Phys. Rev. Lett. 72, 2741 (1994). F. J. Walker, R. A. McKee, Huan-wun Yen, and D. E. Zelmon, Appl. Phys. Lett. 65, p. 1495 (1994).

2. P. K. Tien, R. Ulrich, and R. J. Martin, Appl. Phys. Lett. 14, p. 291 (1969).

3. D. K. Fork, F. Armani-Leplingard, J. J. Kingston, and G.B. Anderson in Thin Films for Integrated Optics, edited by B. W. Wessels, S. R. Marder, and D. M. Walba (Mater. Res. Soc. Proc. 392, Pittsburgh, PA 1995), p. 189.

4. J. H. Harding, Rep. Prog. Phys. 53, p. 1403 (1990).

5. P. W. Tasker and A. M. Stoneham, Proc. Br. Ceram. Soc. 34, p. 1 (1984).

6. Dieter Wolf, Solid State Ionics 75, p. 3 (1995).

7. P. W. Tasker, E. A. Colbourn, and W. C. Mackrodt, J. Am. Ceram. Soc. 68, p. 74 (1985). 
8. P. W. Tasker and D. M. Duffy, Surf. Sci. 137, 91 (1984).

9. D. C. Sayle, S. C. Parker and J. H. Harding, Phil. Mag. A 68, p. 787 (1994).

10. M. H. Yang and C. P. Yang, Phys. Rev. Lett. 73, p. 1809 (1994).

11. R. A. McKee, F. J. Walker, J. R. Conner, E. D. Specht, and D. E. Zelmon, Appl. Phys. Lett. 59, p. 782 (1991).

12. R.A. McKee, F.A. List and F. J. Walker in Multilayers: Synthesis, Properties and Non-Electronic Applications, edited by T. W. Barbee, F. Spaepen, and L. Greer (Mater. Res. Soc. Proc. 103, Pittsburgh, PA 1988), p. 35-39.

13. M. H. Yang and C. P. Flynn, Phys. Rev. Lett. 62, p. 2476 (1989). M. H. Yang and C. P. Flynn, Phys. Rev. B 41, p. 8500 (1990).

14. S. Yadavalli, M. H. Yang and C. P. Flynn, Phys. Rev. B 41, 7961 (1990).

15. M. M. McGibbon, N. D. Browning, M. F. Chisholm, A. J. McGibbon, S. J. Pennycook, V. Ravikumar, and V. P. Dravid, Science 266, p. 102 (1994).

\section{DISCLAIMER}

This report was prepared as an account of work sponsored by an agency of the United States Government. Neither the United States Government nor any agency thereof, nor any of their employees, makes any warranty, express or implied, or assumes any legal liability or responsibility for the accuracy, completeness, or usefulness of any information, apparatus, product, or process disclosed, or represents that its use would not infringe privately owned rights. Reference herein to any specific commercial product, process, or service by trade name, trademark, manufacturer, or otherwise does not necessarily constitute or imply its endorsement, recommendation, or favoring by the United States Government or any agency thereof. The views and opinions of authors expressed herein do not necessarily state or reflect those of the United States Government or any agency thereof. 